\title{
Duration of Tumor-infiltrating Lymphocytes Assessment with Significant Overall Survival Prognostic Value in Locally Advanced Breast Cancer
}

\author{
I Wayan Sudarsa ${ }^{1 *}$ (D), Citra Aryanti ${ }^{2}$ (D) \\ ${ }^{1}$ Division of Surgical Oncology, Department of Surgery, Udayana University, Sanglah General Hospital, Denpasar, Bali, \\ Indonesia; ${ }^{2}$ Department of Surgery, Udayana University, Sanglah General Hospital, Denpasar, Bali, Indonesia
}

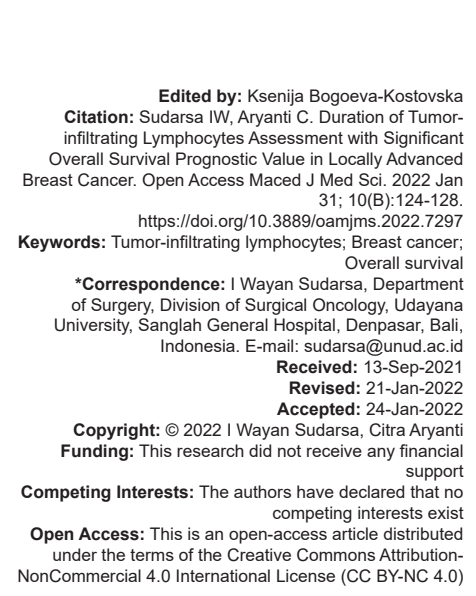

\section{Introduction}

Tumor-infiltrating

lymphocytes

represented the cancer microenvironment as a manifestation of the host immune response against tumor cells [1]. TIL have been studied as a good prognosticator for colorectal cancer [2], non-smallcell lung cancer [3], and breast cancer [4]. High levels of TIL were associated with better chemotherapy response and cancer survival [5]. TIL was a tumor microenvironment parameter that was assessed in almost all histopathological examination.

The modern era has seen a spurt of knowledge regarding TIL timing of collection. The presence of a significant relationship between TIL and prognosis of overall survival was controversial [5], [6]. Theories have concluded that the tumor microenvironment is dynamic [1], [5]. When cancer progresses, the microenvironment will change, thus the outcome of systemic therapy will change as well [7]. Therefore, it is important to assess whether the assessment of TIL at a certain duration still has value as a prognostic factor. Further, we need to determine the duration threshold from TIL assessment until neoadjuvant chemotherapy is still meant to be used as a prognostic factor.

\section{Materials and Methods}

This was a case-control study that included subjects with locally advanced breast cancer in Sanglah General Hospital, registered in Bali's Cancer Registry. The study has been approved by the institutional review board of Udayana University, Denpasar, Bali. The study has been approved by the institutional review board of Udayana University, Denpasar, Bali.

All the data used in this study were obtained from Bali's Cancer Registry and Medical records of Sanglah General Hospital, Bali, Indonesia. We included 
150 random consented subjects in each survived and died group based on the minimum sample calculation. Subjects with immunodeficiency and other malignancy were excluded from this study. Data collected were age, breast cancer subtype, value of TIL, time of the neoadjuvant chemotherapy start, and survival duration. Missing data were obtained from electronic medical records. Hematoxylin and eosin-stained (HES) slide from pretreatment core biopsy specimens were assessed for TIL.

TIL stromal assessment with Hematoxylin and Eosin staining, using an Olympus binocular light microscope conducted by researchers and two specialists in Pathology Laboratory. TIL interpretation was assessed by visual estimation using recommendations by the 2014 International Working Group, TIL was assessed as a percentage of stromal TIL with the denominator being the area of stromal tissue (i.e., the area occupied by mononuclear inflammatory cells compared to total intratumoral stromal tissue). Areas reported are TIL stromal area averages, not just hotspots. TIL are then grouped into low TIL (negative and +1$)$ and high TIL (+2 and +3$)$. The duration of TIL assessment was determined as the time when TIL are being assessed in biopsy specimen to the date of starting chemotherapy, described in days.

Data analysis was done with Statistical Package for the Social Sciences 25.0. Numeric data were described as mean and standard deviation. Categorical data were described as proportions and percentages. After classifying TIL low and high, we performed the diagnostic test with receiver operating curve, searching for Youden Index, sensitivity, specificity, and area under the curve. Kaplan-Meier log-rank analysis was carried out to find the difference between high and low TIL in predicting the overall survival in breast cancer. The significance in this study was $95 \%$ confidence interval.

\section{Results}

In this study, as many as 150 locally advanced breast cancer patients who survived and died in 2016-2020 were analyzed (Table 1). The mean age of subjects in this study was 48.7 (SD 9.3) years with a

Table 1: Characteristics of subjects included in this study

\begin{tabular}{lll}
\hline Characteristic & $\mathrm{N}$ & $\%$ \\
\hline Age & Mean 48.7 years & SD 9.3 \\
Subtype & 52 & 17.3 \\
$\quad$ Luminal A & 62 & 20.7 \\
$\quad$ Luminal B & 66 & 22 \\
$\quad$ Luminal B/HER2 & 102 & 34 \\
$\quad$ HER2 & 18 & 6 \\
$\quad$ TNBC & 84 & 28 \\
TIL & 216 & 72 \\
$\quad$ High & Mean 46.3 days & SD 24.5 \\
$\quad$ Low & 47 months & \\
Duration of TIL assessment & & \\
Median survival & &
\end{tabular}

median survival of 47 months. The majority of patients had the HER2 subtype (34\%), followed by luminal B/ HER2 (22\%), luminal B $(20.7 \%)$, luminal A $(17.3 \%)$, and TNBC (6\%). All patients included in the study were locally advanced breast cancer patients. The biopsy will be performed on patients and biopsy results are usually included with TIL examination. After that, patients will receive neoadjuvant chemotherapy for downstaging before surgery.

The TIL are then grouped into groups with low TIL (negative and +1 ) and high TIL (+2 and +3). In survived group, the proportion of low and high TIL was almost similar, and vice versa. In the death group, $50.5 \%$ of subjects had low TIL levels and in the survived group, $51.2 \%$ of subjects had high TIL. Thus, no significant difference was found between the TIL values and the overall breast cancer survival $(p=0.098)$.

The authors then analyzed the duration of the TIL examination until the neoadjuvant chemotherapy was performed given the dynamicity of the tumor environment. In this study, the average duration of the TIL assessment to neoadjuvant chemotherapy was 46.3 days but a significant standard deviation of 24.5 days. The time when chemotherapy resumed again was $4-446$ days. The reason could be subjects who have not yet been controlled to outpatient after the biopsy, the process of transfer from the primary or secondary hospital where the biopsy was done to the tertiary hospital for chemotherapy or the need for stabilization of conditions such as heart function.

The threshold for how many days the duration of the TIL examination to neoadjuvant chemotherapy can become the overall survival prognostication value was done by using ROC statistics. The comparison was made between the duration of TIL examination of the survived group with high TIL and those unsurvived subjects with low TIL. Comparison between the two groups showed Youden Index as a cut-off point at 30.5 31 days. AUC on ROC was 0.716 with a sensitivity of $64.1 \%$ and specificity of $35.5 \%$ (Figure $1 \mathrm{a}$ and $\mathrm{b}$ ).

Taking 31 days as the cut-off point, we performed further survival analysis for confirmation. In subjects with TIL assessed for more than 31 days, it

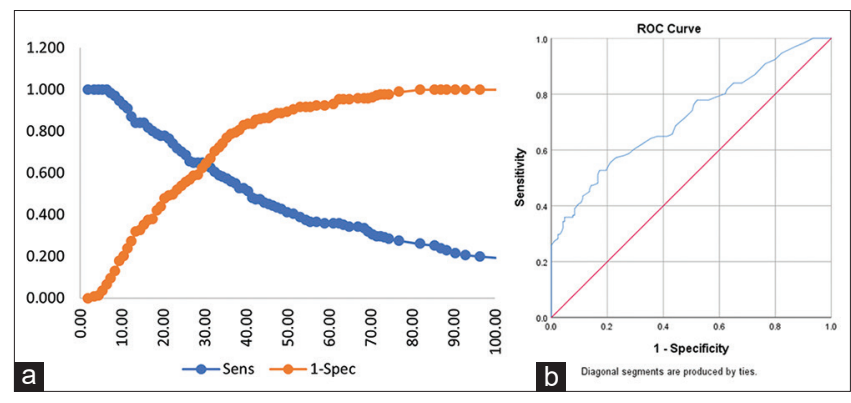

Figure 1: (a) Cut off point of significant tumor-infiltrating lymphocytes assessment duration for prognostication of the overall survival of breast cancer, (b) ROC curve of diagnostic value of tumor-infiltrating lymphocytes assessment duration 
was found that TIL were unable to propose the overall survival of breast cancer ( $p=0.692$; Figure $2 a)$. On the other hand, in subjects with TIL examined for $<31$ days, it was found that TIL could significantly prognosticate the overall survival of breast cancer ( $p=0.005$; Figure $2 b)$.

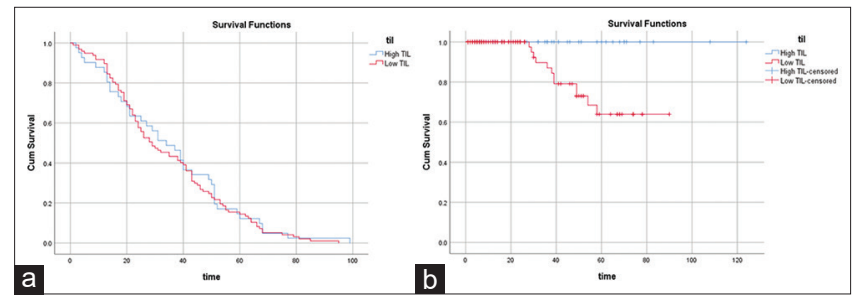

Figure 2: (a) Kaplan-Meier curve of long-term tumor-infiltrating lymphocytes duration assessment and overall survival of breast cancer (log-rank), (b) Kaplan-Meier curve of short-term tumorinfiltrating lymphocytes duration assessment and overall survival of breast cancer (log-rank)

High TIL are associated with better overall survival, and low TIL are associated with poor survival. In the future, it is hoped that clinicians will be more critical in the interpretation of TIL, especially the duration of TIL assessment before neoadjuvant chemotherapy. Examination of TIL for a long time until neoadjuvant chemotherapy cannot be a prognostic factor for the overall survival of breast cancer.

\section{Discussion}

\section{Host-tumor immune response in breast cancer}

Breast cancers encompass a heterogeneous group of tumors. Effective immune surveillance by the host immune system protects the body against cancer [2]. The tumor microenvironment, represented by TIL, is related to tumor therapy response and its progression, the higher the TIL, the better prognosis of cancer [8]. The subpopulation of T-cells played each role in host-tumor immune response where CD8+ served to the destroyed cancer cells and CD4+ served to cause tumor immunosuppression [9]. In invasive breast cancer, the greatest clinical benefits were seen in tumors with $>50 \%$ TIL [10]. Tumor infiltration by lymphocytes thus develops to be a good prognostic factor, reflecting the risk of recurrence or poor overall survival.

\section{The role of TIL}

Found by Aaltomaa in 1990, the role of TIL was studied widely worldwide [11]. Several studies have indicated that TIL were not universal as a prognostic indicator due to the variability of the testing method and complexity of breast cancer molecular subtypes [12]. Currently, researcher showed that the timing of TIL analysis also matters as the microenvironment is dynamically progressing. TIL value was dynamically changed in a subset of time, following the tumor microenvironment and host response to mediators.

The fact that TIL were dynamic that several studies are examining that TIL changes with the administration of systemic therapy [3]. Ladoire et al. observed a higher infiltration of CD8+ TIL and lower infiltration of FOXP3+ TIL after primary systemic therapy. Therefore, it could be that the TIL biopsy examined is neither representative of the survival prognostic nor a predictor of neoadjuvant chemotherapy response because it has been done for a long time and the tumor environment has changed [13]. Besides that, tissue biopsies are small, with a minimal area yielding inadequate information on the position of the cancer tissue.

\section{Duration of TIL assessment to neoadjuvant chemotherapy}

Only one study has been conducted to show the minimal duration of TIL to neoadjuvant chemotherapy that can significantly predict overall and disease-free survival. No study in Indonesia has been conducted. In 239 subjects, Kashiwagi et al. (2019) showed that the timing of TIL assessment during neoadjuvant chemotherapy for breast cancer is more sensitive in the short term group ( $\leq 35$ days) compared to the long term group [14].

To improve the consistency and reproducibility for evaluating TIL in research and clinical practice, we analyzed the threshold of duration from TIL assessment until neoadjuvant chemotherapy is still meant to be used as a prognostic factor. In this study, we found a slightly similar cut-off point of TIL assessment duration, 31 days. AUC on ROC was 0.716 with a sensitivity of $64.1 \%$ and specificity of $35.5 \%$. In subjects with TIL assessed for more than 31 days, it was found that TIL were unable to propose the overall survival of breast cancer $(p=0.692)$. On the other hand, in subjects with TIL examined for $<31$ days, it was found that TIL could significantly prognosticate the overall survival of breast cancer $(p=0.005)$. High TIL are associated with better overall survival and low TIL are associated with poor overall survival [9]. In the future, it is hoped that clinicians will be more critical in the interpretation of TIL, especially the duration of TIL assessment before neoadjuvant chemotherapy. The results of our study showed that examination of TIL with a long period until neoadjuvant chemotherapy could not be a prognostic factor for the overall survival of breast cancer.

The timing theories have not been yet generalized, but the author believed this is due to dynamically changing tumor microenvironment and immune cells competition in peri or intratumoral conditions. Immune cells reaching the tumor bed should pass the extracellular matrix and hostile environment of tumor epithelial [15]. Further, the author also suggested 
differentiating the location of TIL examination, intratumoral or stromal, which has not been routinely done in developing countries.

\section{Strength and limitation of this study}

This study is the first to determine the duration of TIL to neoadjuvant chemotherapy as an essential factor in predicting breast cancer subjects survival in Indonesia. However, the limitation of our study was status of TIL in the biopsy specimens may not reflect that of the entire tumor, only some areas to evaluate the status of TIL subset infiltration. Scanning of the whole slide of the tumor and digital analyses of TIL subsets in each compartment may be the next step to extend this study.

\section{Conclusion}

The duration of TIL to neoadjuvant chemotherapy assessment was important in determining the meaningful TIL value in the prognostication overall survival of breast cancer. The short-term TIL assessment duration (<31 days) predicted well, but not in the long-term assessment duration.

\section{Acknowledgment}

The authors acknowledged the subjects that had participated in this study.

\section{Statement of Ethics}

This study has been approved by the Research Ethical Committee of Faculty of Medicine, Udayana University (973/UN14.2.2.VII.14/LT/2020). Written informed consent was obtained from all the study participants.

\section{References}

1. Fridman WH, Pages F, Salutes-Fridman C, Galon J. The immune contexture in human tumors: Impact on clinical outcome. Nat Rev Cancer. 2012;12(4):298-306.

PMid:22419253
2. Saito $T$, Nishikawa $H$, Wada $H$, Nagano $Y$, Sugiyama $D$, Atarashi $\mathrm{K}$, et al. Two FOXP3(+)CD4(+) T cell subpopulations distinctly control the prognosis of colorectal cancers. Nat Med. 2016;22(6):679-84. https://doi.org/10.1038/nm.4086 PMid:27111280

3. Brambilla E, Le Teuff G, Marguet S, Lantuejoul S, Dunant A, Graziano $\mathrm{S}$, et al. Prognostic effect of tumor lymphocytic infiltration in resectable non-small-cell lung cancer. J Clin Oncol. 2016;34(11):1223-30. https://doi.org/10.1200/ JCO.2015.63.0970

PMid:26834066

4. Perez EA, Ballman KV, Tenner KS, Thompson EA, Badve SS Bailey $\mathrm{S}$, et al. Association of stromal tumor-infiltrating lymphocytes with recurrence-free survival in the N9831 adjuvant trial in patients with early-stage Her2-positive breast cancer. JAMA Oncol. 2016;2(1):56-64. https://doi.org/10.1001/ jamaoncol.2015.3239

PMid:26469139

5. Ibrahim EM, Al-Foheidi ME, Al-Mansour MM, Kazkaz GA. The prognostic value of tumor-infiltrating lymphocytes in triplenegative breast cancer: A meta-analysis. Breast Cancer Res Treatment. 2014;148(3):467-76

PMid:25361613

6. Adams S, Gray RJ, Demaria S, Goldstein L, Perez EA, Shulman LN, et al. Prognostic value of tumor-infiltrating lymphocytes in triple-negative breast cancers from two phase III randomized adjuvant breast cancer trials: ECOG 2197 and ECOG 1199. J Clin Oncol. 2014;32(27):2959-66. https://doi. org/10.1200/JCO.2013.55.0491

\section{PMid:25071121}

7. Ocaña A, Diez-Gónzález L, Adrover E, Fernández-Aramburo A Tumor-infiltrating lymphocytes in breast cancer: Ready for prime time? Clin Oncol. 2015;33:1298-9. https://doi.org/10.1200/ JCO.2014.59.7286

PMid:25753437

8. Mlecnik B, Bindea G, Angell HK, Sasso MS, Obenauf AC Fredriksen $\mathrm{T}$, et al. Functional network pipeline reveals genetic determinants associated with in situ lymphocyte proliferation and survival of cancer patients. Sci Transl Med. 2014;6:228ra237. https://doi.org/10.1126/scitransImed.3007240

PMid:24648340

9. Nosho K, Baba Y, Tanaka N, Shima K, Hayashi M, Meyerhardt J et al. Tumour-infiltrating T-cell subsets, molecular changes in colorectal cancer, and prognosis: Cohort study and literature review. J Pathol. 2010;222(4):350-66. https://doi.org/10.1002/ path. 2774

PMid:20927778

10. Denkert C, Loibl S, Noske A, Roller M, Muller BM, Komor M, et al. Tumor-associated lymphocytes as an independent predictor of response to neoadjuvant chemotherapy in breast cancer. J Clin Oncol. 2010;28(1):105-13. https://doi.org/10.1200/ JCO.2009.23.7370

PMid:19917869

11. Aaltomaa S, Lipponen P, Eskelinen M, Kosma VM, Marin S, Alhava $\mathrm{E}$, et al. Lymphocyte infiltrates as a prognostic variable in female breast cancer. Eur J Cancer. 1990;28(4-5):859-64. https://doi.org/10.1016/0959-8049(92)90134-n

PMid:1524909

12. Mao $Y, Q u Q$, Chen $X$, Huang $O$, Wu J, Shen $K$. The prognostic value of tumor-infiltrating lymphocytes in breast cancer: A systematic review and meta-analysis. PLoS One. 2016;11(4):e0152500. https://doi.org/10.1371/journal. pone. 0152500

PMid:27073890

13. Ladoire S, Mignot G, Dabakuyo S, Arnould L, Apetoh L, Rébé C, 
et al. In situ immune response after neoadjuvant chemotherapy for breast cancer predicts survival. J Pathol. 2011; 224(3):389400. https://doi.org/10.1002/path.2866

PMid:21437909

14. Kashiwagi S, Asano $\mathrm{Y}$, Goto W, Takada K, Takahashi K, Noda S, et al. Use of Tumor-infiltrating lymphocytes (tumor-infiltrating lymphocytes) to predict the treatment response to eribulin chemotherapy in breast cancer.
PLoS One. 2017;12(2):e0170634. https://doi.org/10.1371/ journal.pone.0170634

PMid:28166544

15. Henke E, Nandigama R, Ergun S. Extracellular matrix in the tumor microenvironment and its impact on cancer therapy. Front Mol Biosci. 2020;6:160. https://doi.org/10.3389/ fmolb.2019.00160

PMid:32118030 